\title{
Point-of-care ultrasound (POCUS): unnecessary gadgetry or evidence-based medicine? 鉴
}

\author{
Authors: Nicholas Smallwood ${ }^{A}$ and Martin Dachsel ${ }^{B}$
}

Over the last decade there has been increasing interest and enthusiasm in point-of-care ultrasound (POCUS) as an aide to traditional examination techniques in assessing acutely unwell adult patients. However, it currently remains the domain of a relatively small handful of physicians within the UK. There are numerous reasons for this, notably a lack of training pathways and supervisors but also a lack of understanding of the evidence base behind this imaging modality. This review article aims to explore some of the evidence base behind POCUS for a number of medical pathologies, and where possible compare it to evidenced traditional examination techniques. We discuss the issues around training in bedside ultrasound and recommend a push to integrate POCUS training into internal medicine curricula and support trainers to comprehensively deliver this.

KEYWORDS: Point-of-care ultrasound, POCUS, diagnostics, ultrasound, clinical review

\section{Introduction}

There has been a significant increase in the uptake of point-ofcare ultrasound (POCUS) in the UK over the last two decades. The advent of smaller, more portable machines combined with improved picture quality has brought ultrasound out of the radiology department and onto the wards. In 2002, the National Institute for Clinical Excellence mandated the use of ultrasound in central venous catheter (CVC) insertion, ${ }^{1}$ followed by a National Patient Safety Agency (NPSA) report in 2008 requiring it for pleural procedures ${ }^{2}$ - both of which were in response to compelling evidence for increased safety with ultrasound guided techniques. The practice of focused assessment of sonography in trauma (FAST) scanning has become widespread and the Royal College of Emergency Medicine introduced POCUS as a mandatory component of the emergency medicine curriculum in $2010,{ }^{3}$ with the Respiratory Medicine introducing pleural ultrasound into their curriculum a few years later.

Despite this, there remain significant barriers to the widespread uptake of POCUS within general internal medicine for a number

Authors: ${ }^{\text {A }}$ consultant acute medicine, Surrey and Sussex Healthcare NHS Trust, Surrey, UK; ${ }^{B}$ consultant acute medicine, Surrey and Sussex Healthcare NHS Trust, Surrey, UK of reasons. Part of this is likely to be a lack of understanding of the evidence base which exists for this imaging modality. It is also likely that clinicians place an undue confidence in traditional clinical examination which evidence suggests is often not as robust as thought. This article aims to review the evidence base for POCUS primarily within medical specialties and discuss the future of this imaging modality.

\section{Thoracic ultrasound}

\section{Pleural procedures}

As early as 1990 it was shown in a prospective study of 52 patients that ultrasound guidance versus clinical guidance reduced the incidence of pneumothorax, dry tap or inadequate tap from 14/33 to $0 / 19$ patients. ${ }^{4}$ This was confirmed by a larger retrospective cohort study published in 1991 concerning 342 patients, ${ }^{5}$ which showed a reduction in rates of pneumothorax from $18 \%$ to $3 \%$ with ultrasound guidance. This finding has since been confirmed multiple other times. 6,7

The true incidence of solid organ injury from clinically guided thoracocentesis is infrequently quantified in cohort studies, although it is described. ${ }^{8}$ There are case reports of significant morbidity and mortality associated with clinically guided chest drain insertion, culminating in the 2008 NPSA publication. ${ }^{2}$ It was this influential document which mandated the use of real-time ultrasound guidance for chest drain insertion and has significantly altered clinical practice. It is worth noting that the ' $X$ marks the spot' approach of ultrasound guidance with delayed procedure does not significantly reduce the complication rate and should not be employed. ${ }^{9}$

The accuracy of clinical examination versus ultrasound guidance for determining a safe procedure site has been assessed in a prospective comparative study of 67 patients $^{10}$ and helps to explain some of the observations noted above. This study showed that $15 \%$ of sites determined by clinical examination were unsafe for pleural procedure while ultrasound marked a safe site in $80 \%$ of these cases. Of the $33 \%$ cases where clinical examination could not identify a safe site, ultrasound was able to in $54 \%$ of cases. Overall, ultrasound increased the rate of accurate sites by $26 \%$. Interestingly, this study did not find an association between operator experience and site-marking accuracy.

Overall, the evidence and guidance are compelling that ultrasound guidance (which in many cases will be POCUS) is mandatory prior to pleural procedures for all but pneumothoraces. For completeness, it should be noted that ultrasound has not been 
shown to reduce the incidence of bleeding secondary to intercostal artery injury, as these are not well visualised by ultrasound. It is for this reason that use of the 'safe triangle' for thoracocentesis remains the recommended approach even with ultrasound guidance. $^{9}$

\section{Respiratory failure}

It was traditionally thought that ultrasound as a diagnostic tool for the assessment of respiratory failure would be of limited value due to the aeration of lung interacting with the ultrasound beam and obliterating useful images. However, it has become apparent that as a significant number of pathological lung conditions replace aerated lung with fluids like water, pus and blood, useful clinical information can be gained from examining the lung with ultrasound. Interestingly, it is not just through direct visualisation of lung pathology that diagnoses can be made; many diagnoses will be through understanding the generation and distribution of common sonographic artefacts and this understanding has led to a revolution in bedside lung ultrasound for respiratory failure.

The seminal paper in this regard was published in 2008 by Lichtenstein and Mezière detailing the 'Bedside lung ultrasound in emergency' (BLUE) protocol. ${ }^{11}$ This showed a very good diagnostic accuracy of lung ultrasound for respiratory failure in the most common respiratory conditions and could be achieved with a rapid, protocolised, reproducible approach. These data are summarised in Table 1.

Lichtenstein and Mezière took 260 patients admitted to their intensive care unit with respiratory failure as the primary diagnosis and instituted a protocolised lung and deep venous ultrasound scan taking less than 3 minutes. They were blinded to any history, or prior biochemical or radiological tests and compared their diagnosis to the confirmed final diagnosis generated through traditional means (often computed tomography [CT]); the results are shown in Table 1. There were a number of different 'profiles' generated for each common diagnosis and individually they were not especially sensitive, but their specificity was high. Combining these profiles gave an overall diagnostic accuracy of $90.5 \%$, which is significantly better than both auscultation and chest radiography ${ }^{12}$ and approaches that of $\mathrm{CT}$.

While this single-centre data looks compelling, it is worth remembering that it was conducted on a population of patients requiring critical care and therefore at the severe end of the disease spectrum. A more recent blinded observational evaluation of POCUS for respiratory failure patients admitted from a single
Emergency Department (ED) in Italy has been published, ${ }^{13}$ which may be more generalisable to the 'acute take' cohort of patients seen in general internal medicine. This compared the performance of POCUS-guided diagnosis versus traditional techniques, for both time to diagnosis and concordance between the diagnoses, in 2683 patients. ${ }^{13}$ This study group showed that POCUS significantly reduced the time to diagnosis ( $24+/-10$ min vs $186+/-72$ min), and performed as well as clinical examination plus chest $X$-ray (CXR) for diagnosing acute coronary syndrome, pneumonia, pleural effusion, pericardial effusion, pneumothorax and dyspnoea from other causes. POCUS was more sensitive for heart failure, and less for exacerbations of asthma / chronic obstructive pulmonary disease (COPD) and pulmonary embolism. Overall concordance was good showing a kappa value of 0.71 . This is the largest study looking at undifferentiated patients presenting to the ED, who will generally be less unwell than those in Lichtenstein's seminal study, and may account for some of the differences in diagnostic accuracy.

There is a wealth of other data supporting the use of lung ultrasound for respiratory failure. POCUS has been shown to diagnostically outperform both CXR and auscultation in the intensive care unit setting on more than one occasion, ${ }^{12,14}$ to reduce time to diagnosis and minimise incorrect diagnoses for dyspnoeic patients in the ED, ${ }^{15}$ and the BLUE protocol to shorten the time from admission to treatment in the ED setting. ${ }^{16} \mathrm{~A}$ comparison from Denmark of 320 ED patients with respiratory symptoms showed POCUS improved diagnostic accuracy by $24.3 \%$ (from $63.7 \%$ to $88.0 \%$ ) at 4 hours versus usual care. ${ }^{17}$ The diagnostic accuracy of lung ultrasound in adult patients with pneumonia has been confirmed by a 2014 meta-analysis confirming the sensitivity and specificity to be above $90 \% .{ }^{18}$

The diagnosis and treatment of acute heart failure is another area where lung ultrasound has shown significant promise. The addition of lung ultrasound to traditional management has shown improved diagnostic accuracy over clinical gestalt alone in two large, observational studies. ${ }^{19,20}$ The same studies have in fact confirmed the relatively poor sensitivity of clinical examination alone in diagnosing acute heart failure. These and other data have led to the European Society of Cardiology Acute Cardiovascular Care Association acknowledging the role of lung ultrasound alongside echocardiography in the diagnosis of acute heart failure, ${ }^{21}$ with similar recommendations from the Heart Failure Association of the European Society of Cardiology. ${ }^{22}$

For examples of pathology on thoracic ultrasound, please see Fig 1.

Table 1. Diagnostic accuracies of the BLUE protocol in respiratory failure

\begin{tabular}{|c|c|c|c|c|}
\hline & Sensitivity (\%) & Specificity (\%) & Positive predictive value (\%) & Negative predictive value (\%) \\
\hline Cardiogenic pulmonary oedema & 97 & 95 & 87 & 99 \\
\hline COPD/asthma & 89 & 97 & 93 & 95 \\
\hline Pulmonary embolism & 81 & 99 & 94 & 98 \\
\hline Pneumothorax & 88 & 100 & 100 & 99 \\
\hline Pneumonia & 89 & 94 & 88 & 95 \\
\hline \multicolumn{4}{|c|}{ Overall diagnostic accuracy of the BLUE protocol in these five conditions } & $90.5 \%$ \\
\hline
\end{tabular}




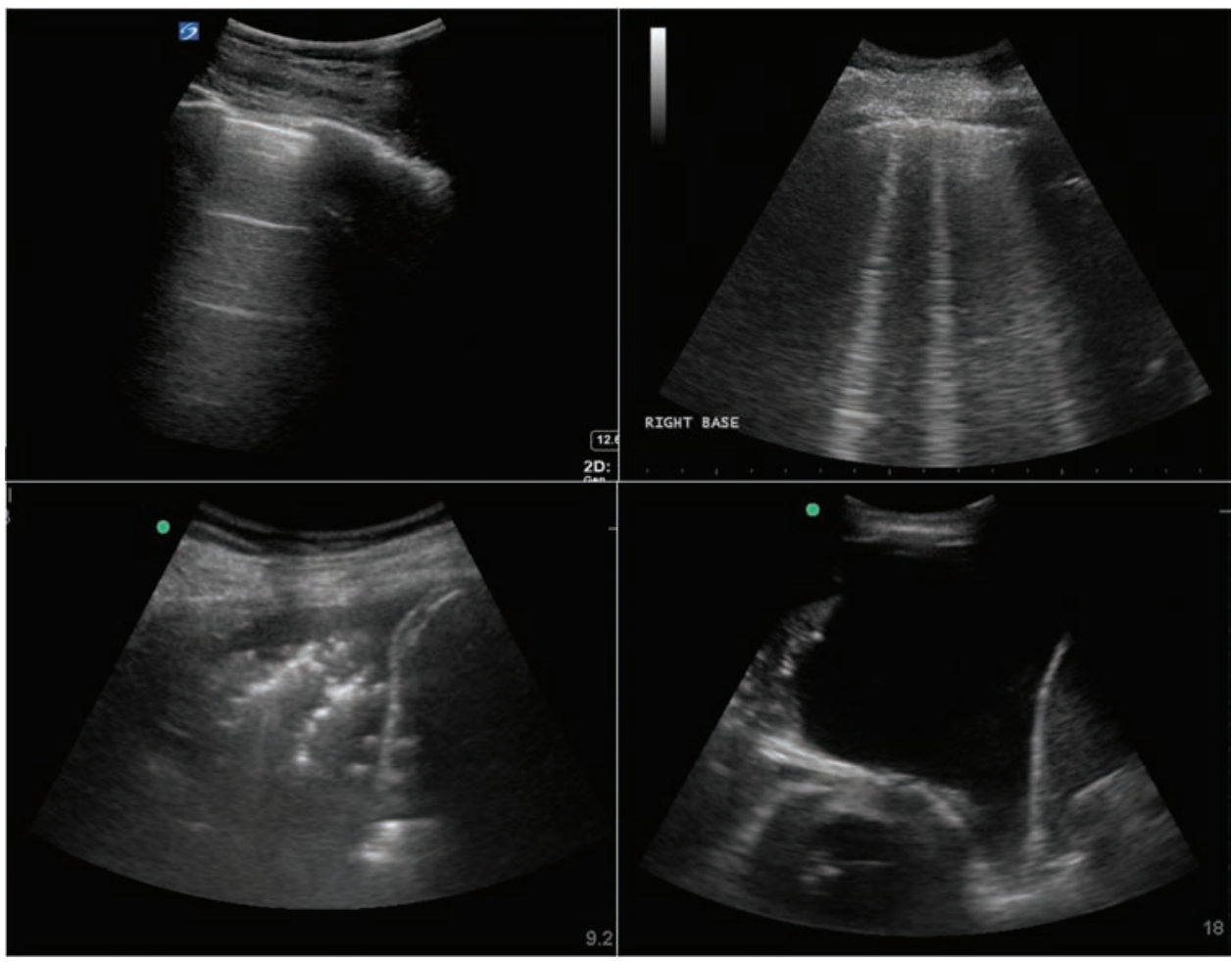

Fig 1. Example images seen during thoracic ultrasound. Clockwise from top left: normal lung appearance with horizontal A line artefacts; vertical B line artefacts seen in a number of conditions; large left pleural effusion with collapsed lung and apex of heart visible; consolidated lung base - the so-called 'shred sign' - with small parapneumonic effusion.

\section{Abdominal ultrasound}

The FAST examination has been commonplace within EDs for at least the last decade and has had a clear role to play in the management of trauma patients. There is, however, an increasing recognition of the limitations of this scan (including low sensitivity and reliability of negative results) $)^{23}$ and it seems likely that increasing availability of $\mathrm{CT}$ will probably negate its role further. A recent Cochrane review failed to demonstrate any benefit of FAST scanning in abdominal or multiple blunt trauma patients, ${ }^{24}$ potentially limiting its future utilisation in this cohort of patients.

There is evidence from an observational cohort study of approximately 70,000 patients undergoing paracentesis that ultrasound reduces the bleeding complication rate by $68 \%{ }^{25}$ A further study involving 100 patients comparing ultrasoundguided versus clinically guided paracentesis showed a significantly improved success rate from $65 \%$ to $95 \%{ }^{26}$

Given that the mainstay of abdominal aortic aneurysm (AAA) monitoring is departmental ultrasound, it is perhaps not surprising that POCUS also shows an impressive sensitivity and specificity for the diagnosis of AAA. A direct comparison of handheld ultrasound device versus conventional duplex ultrasonography for AAA screening showed sensitivity and specificity of $93 \%$ and $97 \%$ respectively. ${ }^{27}$ This compares with sensitivities and specificities of $68 \%$ and $75 \%$ for abdominal palpation alone. ${ }^{28}$

Another area where POCUS may have a role is in the assessment of renal colic in the ED. One of the largest comparator trials to date involving POCUS assessed whether emergency physician (EP) performed ultrasound, radiologist performed ultrasound or CT as the initial investigation in suspected renal colic altered the patient pathway. ${ }^{29} \mathrm{~A}$ total of 2759 patients were randomised and the investigators found that initial ultrasound (either EP or radiologist) was associated with lower cumulative radiation exposure than initial CT. This was achieved without significant differences in highrisk diagnoses with complications, serious adverse events, pain scores, return emergency department visits or hospitalisation.

Examples of pathology within the abdomen can be seen in Fig 2.

\section{Cardiac arrest}

The largest study in the use of POCUS in cardiac arrest was a non-randomised, prospective, protocol driven observational study across 20 centres in the USA and Canada. ${ }^{30}$ They took 793 patients in 'non-shockable' cardiac arrest and performed ultrasound at the beginning and end of the resuscitation process. Patients were analysed according to the presence or absence of sonographic cardiac activity. One-third of patients (33\%) were found to have cardiac activity on ultrasound at the point of cardiac arrest. This was associated with an increased rate of return of spontaneous circulation (ROSC; $51 \%$ achieved ROSC in this group) and importantly a significantly increased survival to hospital discharge (3.8\% vs $0.6 \%$ ). The absence of cardiac activity was associated with a significant reduction in ROSC (14.3\%) and a very poor survival to hospital discharge $(0.6 \%)$. Therefore, it was concluded that POCUS during cardiac arrest could help stratify early a cohort of patients in whom the prognosis was exceptionally poor, which may help influence the decision to continue. Where the aetiology of arrest was found to be pericardial tamponade, the survival to discharge in the POCUS group improved to $15.4 \%$. Given the difficulty in diagnosing tamponade clinically in the arrested patient, this is perhaps not surprising but is hugely encouraging in this small subset of patients. 
Fig 2. Example images seen during abdominal ultrasound. Clockwise from top left: normal liver and kidney; ascites around liver tip and in Morrison's pouch; thick-walled gallbladder with pericholecystic fluid and a stone in the neck; severe hydronephrosis with dilated ureter and renal cortical thinning.
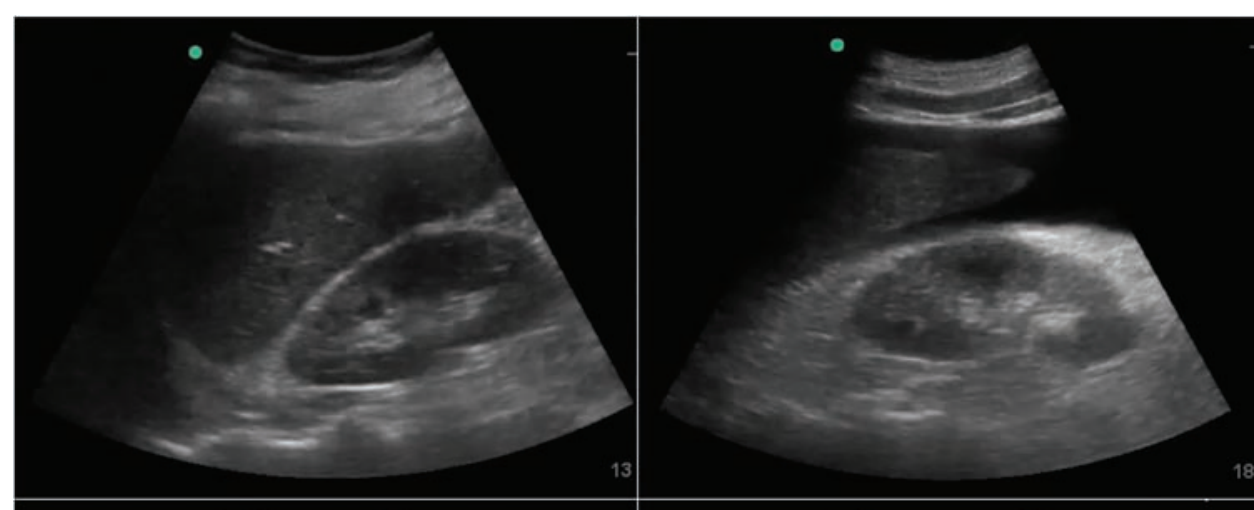

-

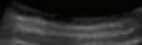

Saris

Q
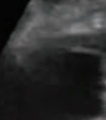

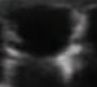

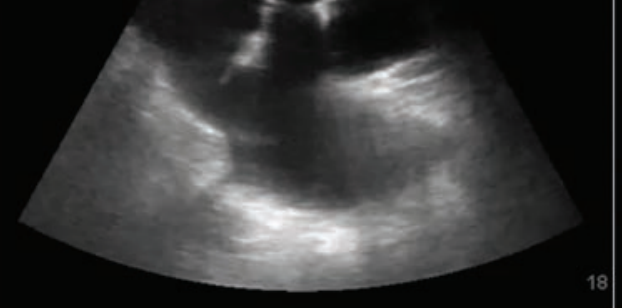

f

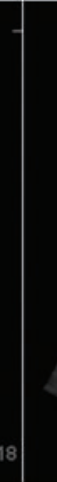

The data are not entirely positive regarding POCUS in cardiac arrest. A recent prospective cohort study of 23 patients undergoing resuscitation in a single centre ED found that the use of POCUS during resuscitation significantly increased the pulse check duration from 13 seconds to 21 seconds. ${ }^{31}$ This study was not designed to look at subsequent outcomes but highlights the importance of minimising delays associated with POCUS during cardiac arrest.

The recently published consensus guidelines from the International Federation for Emergency Medicine stress the importance of performing the ultrasound during the pulse check, saving the clip and reviewing it during the next cycle to avoid increased pulse check duration. ${ }^{32}$

\section{Focused echocardiography and shock}

Care for patients with shock is challenging. The first strong evidence for the usefulness of POCUS was published early in the last decade. A randomised controlled trial involving $184 \mathrm{ED}$ patients with undifferentiated hypotension published in 2004 showed that immediate, goal-directed ultrasound increased the rate of correct diagnosis of shock aetiology from $50 \%$ to $80 \%$ (number needed to treat of 3.3). ${ }^{33}$ The average scan time was $5.8 \mathrm{~min}$.

Bedside echocardiography is a key element of shock ultrasound. There are a number of protocols for the diagnosis of shock aetiology. In 2010, the RUSH (Rapid ultrasound in shock and hypotension) protocol was published. ${ }^{34}$ This combined echocardiography, lung ultrasound, inferior vena cava (IVC) / aorta, pleura and peritoneal windows, and can help discriminate between hypovolaemic, obstructive, cardiogenic and distributive shock. Specific treatments for different shock aetiologies can be started rapidly. Additionally, serial examinations to monitor treatment effects can be performed multiple times without repeated irradiation exposure. Particularly for patients with distributive shock the response to fluid boluses can be monitored (looking for early signs of fluid overload on chest ultrasound) and the treatment strategy can be altered accordingly (early vasopressors / inotropes if signs of leakage into lung interstitium or poor cardiac function). The evidence supporting the use of focused echocardiography in shock is comprehensive and has been synthesised into a consensus document endorsed by the American Society of Echocardiography and the British Society for Echocardiography. ${ }^{35}$

\section{Discussion}

Point-of-care ultrasound is rapidly gaining an evidence base to support its use as an aide to traditional management techniques in the acutely unwell adult patient. There is an increasing body of evidence showing it improves safety during medical procedures and can aid diagnostic accuracy in a number of medical presentations, as outlined above. There is little evidence to suggest harms associated with this imaging modality.

That is not to say, however, that there aren't potential issues. Much of the data outlined here is acquired from single centres and must be translated into multicentre, randomised trials in order to truly understand the effects of POCUS. Similarly, much of the current data pertains to the critical care population, who are by definition at the most severe end of the pathological spectrum; this may mean that the diagnostic accuracies reported in some of this work are not truly generalisable to those patients seen 
routinely in general internal medicine. It is this factor that may help explain the reduced diagnostic accuracy seen with POCUS in respiratory failure in an Italian ED patient cohort ${ }^{13}$ versus a French critical care population ${ }^{12}$. More studies are needed in unselected adult medical patients to clarify these differences.

It can also be argued that although much of this evidence highlights an improved diagnostic accuracy and reduced time to diagnosis, there is no current evidence to highlight a difference in patient outcomes. Hopefully this data will accumulate with time, but it is worth noting that many current standard imaging modalities also do not carry evidence of improved patient outcomes, but they are assumed from the obvious benefits of making the correct diagnosis rapidly. Therefore, it seems reasonable to assume that the diagnostic benefits of POCUS may translate into improved patient outcomes. There is certainly evidence to suggest that patients gain satisfaction from POCUS and it adds to the doctor-patient relationship. ${ }^{36,37}$

Perhaps the biggest barrier to the widespread uptake of POCUS, particularly in the UK, has been the lack of available trainers and suitable training curricula. ${ }^{38,39}$ The Royal College of Radiologists have published ultrasound training standards for medical and surgical specialties, ${ }^{40}$ but these do not include any detail on respiratory failure and do not follow the more binary approach that POCUS encourages. In 2016, the Society for Acute Medicine published the first POCUS curriculum within the UK designed for physicians (Focused acute medicine ultrasound; FAMUS) ${ }^{41}$, which covers a number of areas considered to be important in the management of the acutely unwell patient. Similar curricula are being or have been developed in a number of other countries for internal medicine, with a Canadian group recently publishing their recommendations. ${ }^{42}$ Having a curriculum in place is important to standardise training in this emerging field; however, establishing it will need an adequate number of trainers. This has been seen in respiratory medicine where despite thoracic ultrasound being a mandatory part of the curriculum, trainees have reported difficulties in getting formally signed off. ${ }^{43}$ In order to overcome this barrier for POCUS, there would have to be a concerted effort to develop it as an integral part of the internal medicine training curriculum, and invest time and money in supporting a cohort of trainers to deliver that training. There is certainly an appetite among acute internal medicine trainees to learn POCUS as a core part of their curriculum. ${ }^{44}$

In summary, there is a solid evidence base for POCUS improving traditional examination techniques in the diagnosis and management of the acutely unwell medical patient and it is expanding rapidly. The increasing availability of ultrasound machines coupled with an enthusiasm from trainees to learn bedside ultrasound means that national training committees need to start considering how it can be routinely integrated into medical curricula. In addition, there needs to be a commitment to support the development of trainers with both time and resources to ensure adequate training can actually occur, as opposed to the ad hoc approach that currently exists which relies primarily on the good will of trainers to deliver training to a select few. The authors fully support the integration of POCUS into the acute internal medicine and/or internal medicine curricula currently being developed.

\section{Conflicts of interest}

The authors are both members of the FAMUS working group.

\section{References}

1 National Institute for Clinical Excellence. Guidance on the use of ultrasound locating devices for placing central venous catheters. London: NICE, 2002.

2 National Patient Safety Association. Chest drains: risks associated with the insertion of chest drains. London: NPSA, 2008.

3 Royal College of Emergency Medicine. Ultrasound Training. www. rcem.ac.uk/RCEM/Exams_Training/UK_Trainees/Ultrasound_ Training/RCEM/Exams_Training/UK_Trainees/Ultrasound_Training [Accessed 21 September 2017].

4 Grogan DR, Irwin RS, Channick et al. Complications associated with thoracentesis. A prospective, randomized study comparing three different methods. Arch Intern Med 1990;150:873-7.

5 Raptopoulos V, Davis LM, Lee G et al. Factors affecting the development of pneumothorax associated with thoracentesis. Am J Roentgenol 1991;156:917-20.

6 Barnes TW, Morgenthaler TI, Olson EJ et al. Sonographically guided thoracentesis and rate of pneumothorax. J Clin Ultrasound 2005;33:442-6.

7 Gordon CE, Feller-Kopman D, Balk EM, Smetana GW. Pneumothorax following thoracentesis: a systematic review and meta-analysis. Arch Intern Med 2010;170:332-9.

8 Seneff MG, Corwin RW, Gold LH, Irwin RS. Complications associated with thoracocentesis. Chest 1986;90:97-100.

9 Havelock T, Teoh R, Laws D, Gleeson F on behalf of the BTS Pleural Disease Guideline Group. Pleural procedures and thoracic ultrasound: British Thoracic Society pleural disease guideline 2010. Thorax 2010;65(Suppl 2):ii61-ii76.

10 Diacon AH, Brutsche MH, Solèr M. Accuracy of pleural puncture sites: a prospective comparison of clinical examination with ultrasound. Chest 2003;123:436-41.

11 Lichtenstein DA, Mezière GA. Relevance of lung ultrasound in the diagnosis of acute respiratory failure: the BLUE protocol. Chest 2008;134:117-25.

12 Lichtenstein D, Goldstein I, Mourgeon E et al. Comparative diagnostic performances of auscultation, chest radiography, and lung ultrasonography in acute respiratory distress syndrome. Anesthesiology 2004;100:9-15.

13 Zanobetti M, Scorpiniti M, Gigli C, Nazerian P, Vanni S. Point-of-care ultrasonography for evaluation of acute dyspnea in the ED. Chest 2017;151:1295-301.

14 Inglis AJ, Nalos M, Sue KH et al. Bedside lung ultrasound, mobile radiography and physical examination: a comparative analysis of diagnostic tools in the critically ill. Crit Care Resusc 2016;18:116-24.

15 Pirozzi C, Numis FG, Pagano A et al. Immediate versus delayed integrated point-of-care ultrasonography to manage acute dyspnea in the emergency department. Crit Ultrasound J 2014;6:5.

16 Seyedhosseini J, Bashizadeh-Fakhar G, Farzaneh S, Momeni M, Karimialavijeh E. The impact of the BLUE protocol ultrasonography on the time taken to treat acute respiratory distress in the ED. Am J Emerg Med 2017;35:1815-8.

17 Laursen CB, Sloth E, Lassen AT et al. Point-of-care ultrasonography in patients admitted with respiratory symptoms: a single-blind, randomised controlled trial. Lancet Respir Med 2014;2:638-46.

18 Chavez MA, Shams N, Ellington LE et al. Lung ultrasound for the diagnosis of pneumonia in adults: a systematic review and metaanalysis. Respir Res 2014;15:50.

19 Russell FM, Ehrman RR, Cosby K et al. Diagnosing acute heart failure in patients with undifferentiated dyspnea: a lung and cardiac ultrasound (LuCUS) protocol. Acad Emerg Med 2015;22:182-91.

20 Pivetta E, Goffi A, Lupia E et al. Lung ultrasound-implemented diagnosis of acute decompensated heart failure in the ED. Chest 2015;148:202-10.

21 Price S, Platz E, Cullen L et al. Expert consensus document: Echocardiography and lung ultrasonography for the assessment and management of acute heart failure. Nat Rev Cardiol 2017;14:427-40. 
22 Mebazaa A, Yilmaz MB, Levy P et al. Recommendations on pre-hospital and early hospital management of acute heart failure: a consensus paper from the Heart Failure Association of the European Society of Cardiology, the European Society of Emergency Medicine and the Society of Academic Emergency Medicine. Eur ] Heart Fail 2015:17:544-58.

23 Smith J. Focused assessment with sonography in trauma (FAST): should its role be reconsidered? Postgrad Med J 2010;86:285-91.

24 Stengel D, Rademacher G, Ekkernkamp A, Güthoff C, Mutze S. Emergency ultrasound-based algorithms for diagnosing blunt abdominal trauma. Cochrane Database of Syst Rev 2015;17:CD004446.

25 Mercaldi C], Lanes SF. Ultrasound guidance decreases complications and improves the cost of care among patients undergoing thoracentesis and paracentesis. Chest 2013;143:532-8.

26 Nazeer SR, Dewbre H, Miller AH. Ultrasound-assisted paracentesis performed by emergency physicians vs the traditional technique: a prospective, randomized study. Am J Emerg Med 2005;23:363-7.

27 Lin PH, Bush RL, McCoy SA et al. A prospective study of a handheld ultrasound device in abdominal aortic aneurysm evaluation. Am J Surg 2003;186:455-9.

28 Lederle FA, Simel DL. The rational clinical examination. Does this patient have abdominal aortic aneurysm? JAMA 1999;281:77-82.

29 Smith-Bindman R, Aubin C, Bailitz ] et al. Ultrasonography versus computed tomography for suspected nephrolithiasis. N Engl ] Med 2014:371:1100-10.

30 Gaspari R, Weekes A, Adhikari S et al. Emergency department point-of-care ultrasound in out-of-hospital and in-ED cardiac arrest. Resuscitation 2016;109:33-9.

31 Huis In 't Veld MA, Allison MG, Bostick DS et al. Ultrasound use during cardiopulmonary resuscitation is associated with delays in chest compressions. Resuscitation 2017;119:95-8.

32 Atkinson P, Bowra J, Milne J et al. International Federation for Emergency Medicine Consensus Statement: Sonography in hypotension and cardiac arrest (SHoC): An international consensus on the use of point of care ultrasound for undifferentiated hypotension and during cardiac arrest. CJEM 2016;21:1-12.

33 Jones AE, Tayal VS, Sullivan DM, Kline JA. Randomized, controlled trial of immediate versus delayed goal-directed ultrasound to identify the cause of nontraumatic hypotension in emergency department patients. Crit Care Med 2004;32:1703-8.
34 Perera P, Mailhot T, Riley D, Mandavia D. The RUSH exam: rapid ultrasound in shock in the evaluation of the critically ill. Emerg Med Clin North Am 2010:28:29-56.

35 Via G, Hussain A, Wells M, Reardon R, ElBarbary M et al. International evidence-based recommendations for focused cardiac ultrasound. J Am Soc Echocardiogr 2014;27:683.e1-683.e33

36 Howard ZD, Noble VE, Marill KA et al. Bedside ultrasound maximizes patient satisfaction. J Emerg Med 2014;46:46-53.

37 Claret PG, Bobbia X, Le Roux S et al. Point-of-care ultrasonography at the ED maximizes patient confidence in emergency physicians. Am J Emerg Med 2016;34:657-9.

38 Jaques A, Walden A, Pettipher A. Focused intensive care echocardiography: lots of participation, not much accreditation. J Intensive Care Soc 2017:18:73.

39 Smallwood N, Matsa R, Lawrenson P, Messenger J, Walden A. A UK wide survey on attitudes to point of care ultrasound training amongst clinicians working on the Acute Medical Unit. Acute Med 2016;14:159-64.

40 Royal College of Radiologists. Ultrasound training recommendations for medical and surgical specialties: third edition. London: RCR, 2017.

41 Smallwood N, Dachsel M, Matsa R, Tabiowo E, Walden A. Focused acute medicine ultrasound (FAMUS) - point of care ultrasound for the acute medical unit. Acute Med 2016:15:193-6.

42 Ma IWY, Arishenkoff S, Wiseman J et al. Internal medicine pointof-care ultrasound curriculum: consensus recommendations from the canadian internal medicine ultrasound (CIMUS) group. J Gen Intern Med 2017;32:1052-7.

43 Sutherland T], Dwarakanath A, White H, Kastelik JA. UK national survey of thoracic ultrasound in respiratory registrars. Clin Med 2013:13:370-3

44 Smallwood N, Conway N. Acute internal medicine trainee survey 2015. Acute Med 2015;14:73-7.

Address for correspondence: Dr Nicholas Smallwood, Surrey and Sussex Healthcare NHS Trust, Department of Acute Medicine, East Surrey Hospital, Canada Avenue, Redhill RH1 5RH, UK.

Email: nicholas.smallwood@nhs.net 